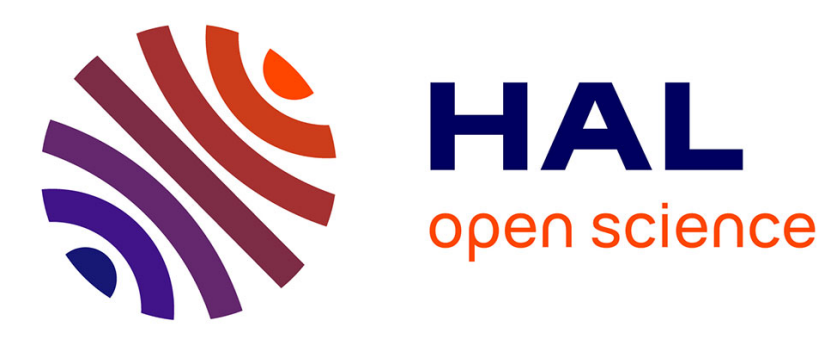

\title{
Dimers of polycyclic aromatic hydrocarbons: the missing pieces in the soot formation process
}

Xavier Mercier, Olivier Carrivain, Cornélia Irimiea, Alessandro Faccinetto, Eric Therssen

\section{- To cite this version:}

Xavier Mercier, Olivier Carrivain, Cornélia Irimiea, Alessandro Faccinetto, Eric Therssen. Dimers of polycyclic aromatic hydrocarbons: the missing pieces in the soot formation process. Physical Chemistry Chemical Physics, 2019, 21 (16), pp.8282-8294. 10.1039/c9cp00394k . hal-02299005

\section{HAL Id: hal-02299005 https://hal.science/hal-02299005}

Submitted on 27 Sep 2019

HAL is a multi-disciplinary open access archive for the deposit and dissemination of scientific research documents, whether they are published or not. The documents may come from teaching and research institutions in France or abroad, or from public or private research centers.
L'archive ouverte pluridisciplinaire HAL, est destinée au dépôt et à la diffusion de documents scientifiques de niveau recherche, publiés ou non, émanant des établissements d'enseignement et de recherche français ou étrangers, des laboratoires publics ou privés. 


\section{Title: Dimers of Polycyclic Aromatic Hydrocarbons: the Missing}

\section{Pieces in the Soot Formation Process}

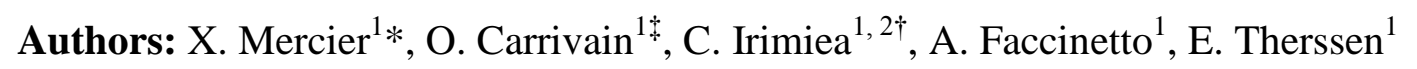

\section{Affiliations:}

${ }^{1}$ Université Lille, CNRS, UMR 8522 - PC2A - Physicochimie des Processus de Combustion et de l'Atmosphère, F-59000 Lille, France,

${ }^{2}$ Université Lille, CNRS, UMR 8522 - PhLAM - Physique des Lasers, Atomes et Molecules, F-59000 Lille, France

Curent affiliations: ${ }^{*}$ LEMTA, Université de Lorraine CNRS, Vandoeuvre-lès-Nancy, 54518, France † DMPE, ONERA, Université Paris Saclay F-91123 Palaiseau - France

*Correspondence to: Xavier Mercier (xavier.mercier@univ-lille.fr)

Tel: ++ 33320434804

\section{Abstract:}

The soot nucleation process, defined as the transition from molecular precursors to condensed matter, is the less understood step in the whole soot formation process. The possibility that polycyclic aromatic hydrocarbon (PAH) dimers, especially those involving moderate-sized PAHs, can play a major role in soot nucleation is a very controversial issue. Although PAH dimers have often been considered as potential soot precursors, their formation is not thermodynamically favored at typical flame temperature, their binding energies being considered too weak to allow them to survive in this environment. Hereby, we report experimental evidence highlighting the existence of PAH dimers in the proximity of the soot 
27 nucleation region of a methane laminar diffusion flame that give strong evidence for the 28 nucleation process to be kinetically rather than thermodynamically controlled.

29

30 Keywords: soot nucleation, polycyclic aromatic hydrocarbons (PAHs), dimers of PAHs, laser 31 induced fluorescence (LIF)

32

33

34 


\section{Introduction:}

Soot emissions are a major source of natural and anthropogenic atmospheric pollution well known for having a measurable impact on human health and environment ${ }^{1}$. It is common knowledge that soot is formed during the incomplete combustion of hydrocarbons. However, a detailed and comprehensive understanding of the fundamental mechanisms of soot formation has not been reached yet. Over the last decades, numerous works focused on the determination of the chemical mechanisms governing soot formation through the identification of the involved species ${ }^{2,3}$. Some key steps of these mechanisms have been established that highlighted the central role of polycyclic aromatic hydrocarbons (PAHs). PAHs formed during the combustion process are mainly issued from reaction pathways involving benzene according to a major chemical growth process known as HACA mechanism ${ }^{4}$, and are well known to contribute to the inception of soot particles. However, the soot nucleation process, corresponding to the transition from gaseous molecular precursors to condensed matter, is still mostly unknown ${ }^{5}$. Many kinetic models that simulate soot formation invoke the dimerization of small PAHs up to 4-5 aromatic rings as the elementary building block of soot particles. Nevertheless, this hypothesis is generally considered as a mere numerical tool rather than realistically mirroring a physical phenomenon. The physical reality of dimerization is usually ruled out because of the weakness of PAH-PAH interactions, along with detailed calculations showing dimers of moderate-sized PAHs to be thermodynamically instable at flame temperature ${ }^{5}$. From theoretical calculations based on thermodynamic considerations, only PAHs as large as ovalene $\left(\mathrm{C}_{32} \mathrm{H}_{14}\right)$ or circumcoronene $\left(\mathrm{C}_{54} \mathrm{H}_{18}\right)$ may lead to the formation of dimers capable to survive at flame temperature ${ }^{5,6}$. However, the amount of soot particles measured in sooting flames is several orders of magnitude higher than typical concentrations of such large PAHs ${ }^{7}$. The implication of dimers of PAHs of this size in the soot nucleation process appears 
60 therefore highly unlikely ${ }^{5,7}$. Other hypotheses relying on the chemical coalescence of PAHs

61 into cross-linked three-dimensional structures has also been proposed ${ }^{8}$. This pathway requires numerous $\mathrm{H}$ atoms to generate aryl radicals by hydrogen abstraction, capable to react with aromatic molecules to form such three-dimensional structures. However, this mechanism has also been proven inadequate to explain the nucleation at low temperature where $\mathrm{H}$ abstraction is limited as well as the persistent nucleation observed into the burnt gases of premixed 66 flames ${ }^{9,10}$.

Primary soot particles are organized in a quasi-amorphous central core surrounded by concentric graphene-like layers ${ }^{11,12}$. Nascent soot particles (NSPs) are less carbonized than mature soot and show a significantly different structure characterized by clusters with randomly distributed orientation inside the core, and can be as small as $1-3 \mathrm{~nm}^{13}$. This size has been shown to be consistent with clusters of PAHs containing up to 5 aromatic rings and 20 carbon atoms ${ }^{14,15}$. Finally, NSPs are characterized by lower density, closer to the density of moderate-sized PAHs as pyrene $\left(1.27 \mathrm{~g} / \mathrm{cm}^{3}\right)$ rather than mature soot $\left(1.80 \mathrm{~g} / \mathrm{cm}^{3}\right)^{16}$, indicating that a graphitization/dehydrogenation process takes place during the soot growth process.

These data strengthen the idea that clusters of moderate-sized PAHs may be involved in the nucleation process, despite the unfavorable thermodynamic conditions for their formation at flame temperature. Herdman and Miller ${ }^{17}$ used density functional theory (DFT) calculations to estimate the intermolecular potentials of PAH clusters to define the size threshold beyond which PAH dimers can be formed in flames. Calculated data ${ }^{17}$ suggest that 81 the binding energy of many pericondensed PAHs, including moderate-sized PAHs, may be 82 large enough to form dimers at flame temperature. However, Wang ${ }^{5}$ revisited these conclusions by considering the entropy resistance to the binding that can prevent dimerization even if the binding energies are large enough. Wang ${ }^{5}$ thus defined an equilibrium constant for 
the dimerization and concluded that only PAHs as large as circumcoronene $\left(\mathrm{C}_{54} \mathrm{H}_{18}\right)$ can survive in a flame environment. Nevertheless, in this approach, dimerization was always considered to be thermodynamically controlled.

To allow the formation of dimers of moderate-sized PAHs in flames, an emerging theory suggests that nucleation might be governed by kinetics rather than thermodynamics. Schuetz and Frenklach ${ }^{18}$ first considered this hypothesis. Notably, they showed by nonequilibrium molecular dynamics simulations that the lifetime of pyrene dimers might be greatly enhanced by the deposition of energy into internal rotations in the colliding pair of pyrene under flame conditions. In this framework, dimers may survive long enough to evolve into NSPs. This idea was elaborated in kinetic models of soot formation in recent papers. Notably, Eaves et al. ${ }^{15,19}$ introduced the reversibility of the nucleation based on the dimerization of PAHs sizing from naphthalene up to benzo(a)pyrene. Aubagnac-Karkar et al. ${ }^{20}$ used the hypotheses of the reversibility of the nucleation and pyrene dimerization to successfully reproduce soot volume fraction profiles experimentally measured in five premixed flames characterized by different equivalence ratio, fuels and pressure conditions leading to a variation of seven orders of magnitude between the numerical results and the experimentally obtained soot volume fractions. More recently, Kholghy et al. ${ }^{21}$ used the dimerization of small to moderate-sized PAHs in their kinetic model, that also considers the reversible formation of PAH clusters by van der Waals forces. In particular, they advocate dimer stabilization through the formation of covalent carbon-carbon bonds following two hydrogen abstractions from the monomers in a dimer. Both approaches show very good agreement of the calculated and experimental soot volume fraction profiles for different flames. They also show that only small to moderate-sized PAHs are sufficient to model soot volume fraction profiles in agreement with the experimental data. Finally, in a very recent paper, Johansson et al. ${ }^{22}$ highlighted that dimers of PAHs may be stabilized into covalently 
110 bound cluster at high temperature according to a rapid radical-driven hydrocarbon clustering

111 mechanism and lead to the formation of soot particles. This work provides another consistent 112 explanation for the existence and the potential role of dimers of PAHs in the soot nucleation 113 process.

114 However, so far no clear experimental evidence on the formation of dimers of 115 moderate-sized PAHs has ever been given in the literature. Such evidence is therefore highly 116 needed, given their potential role as cornerstones of the soot nucleation processes. The 117 absence of experimental evidences most likely comes from the practical difficulty of 118 preserving the dimers during the preparation of samples for online or ex-situ measurements, 119 thus probably precluding the use of techniques as gas chromatography (GC) or mass 120 spectrometry (MS) that are otherwise widely used for the detection of PAHs in flames.

121 Because of their short lifetime in flames, dimers of PAHs likely require in-situ techniques as 122 laser diagnostics to be detected. The spectroscopy of dimers of PAHs has been extensively studied in condensed phase

$124{ }^{23}$ and supersonic expansions ${ }^{24}$. The UV excitation of these species leads to the formation of 125 excimers (dimers in an excited state) characterized by intense, broad and featureless 126 fluorescence emission in the visible spectral range. Dimers of PAHs share electronic excitation transitions with the corresponding monomers, but their emission spectra are systematically shifted toward higher wavelengths ${ }^{25}$. The observation of broadband and unstructured visible emission spectra under UV and

130 visible excitation in sooting flames is not new. Early mentions date back to the $1980 \mathrm{~s}^{26,27}$ and 131 were first attributed to PAHs like acenaphthylene and fluoranthene. In a previous work, we 132 explored the possibility of excitation of such moderate-sized PAHs to explain the origin of 133 this visible fluorescence emission ${ }^{28}$. However, acenaphthylene is characterized by a very 134 weak fluorescence quantum yield ${ }^{29}$ while fluoranthene does not fluoresce far in the visible as 
135 we show below in this paper. At present, the most commonly accepted explanation for these

136 experimental emission spectra measured in the visible range relies on the idea that larger

137 PAHs are characterized by fluorescence emission progressively extended toward higher

138 wavelengths ${ }^{30}$. Hence, fluorescence above $450 \mathrm{~nm}$ is generally attributed to PAHs constituted

139 of more than 4 aromatic rings. In this paper, we demonstrate that this hypothesis is also very

140 unlikely. Finally, a few papers in the literature exist supporting the idea that these signals

141 could be issued from the fluorescence of dimers of PAHs ${ }^{31,32}$. Miller et al. provided

142 convincing insights by DFT calculation that the broadband visible emission can be attributed

143 to aromatic excimers ${ }^{17,31}$. More recently, Sirignano et al. carried out a series of experiments

144 in a sooting flame also suggesting the possibility that the visible emission fluorescence might

145 be characteristic of large PAHs and small clusters (dimers or trimers) of PAHs ${ }^{32}$.

146 Our present work aims to clarify the origin of the intense visible emission spectra

147 observed in sooting flames under laser excitation and consequently prove the existence and

148 the critical implication of dimers of moderate sized PAHs in the nucleation of NSPs. This

149 demonstration is based on the analysis of laser induced fluorescence (LIF) experiments

150 performed in a co-flow laminar diffusion methane-air flame. The analysis of the emission

151 fluorescence has been provided thanks to a simple spectroscopic model capable to simulate

152 these spectra. This model relies on the use of a spectral database, described below, enabling

153 the simulation of the measured fluorescence spectra measured from the flame. This analysis

154 clearly reveals two kinds of fluorescent species that are attributed to PAH monomers and

155 dimers, and discriminated by their different spectroscopic signatures.

\section{Experimental Setup}

LIF measurements have been carried out in the centerline of a methane/air co-flow 
160 induced incandescence (LII) to begin around $60 \mathrm{~mm}$ height above the burner (HAB) ${ }^{33}$. The

161 experimental configuration of the LIF measurements is shown in fig. 1. The flame was setup on a modified McKenna burner, where the porous plug has been replaced with a central tube $(\mathrm{d}=10.25 \mathrm{~mm})$ which allows the injection of methane. The outer air flow tube $(\mathrm{d}=88 \mathrm{~mm})$

164 produces a homogenous air shield on account of a layer of glass beads placed between the 165 central injector and the burner edges. A $120 \mathrm{~mm}$ height non-smoking laminar diffusion flame 166 is stabilized using $0.52 \mathrm{~L} \mathrm{~min}^{-1}$ of $\mathrm{CH}_{4}$ and $87 \mathrm{~L} \mathrm{~min}^{-1}$ of air. The burner and flame configuration are described in detail in Irimiea et al. ${ }^{34}$.

The excitation source consisted of a Nd:YAG laser (Quanta-ray, Spectra Physics), generating laser pulses at 1064, 532 and $355 \mathrm{~nm}$ (pulse length $6 \mathrm{ns)} \mathrm{which} \mathrm{are} \mathrm{mixed} \mathrm{and} \mathrm{used}$ to pump an OPO (premiScan-ULD/240, GWU-Lasertechnik) and provide wavelengths tunable from 213 to $532 \mathrm{~nm}$. LIF measurements have been performed at 213.5, 282, 405, 488 and 532 $\mathrm{nm}$ excitation wavelengths. After the laser output, the laser beam was slightly focused at the center of the diffusion methane/air flame using a spherical UV lens ( $\mathrm{f}=1000 \mathrm{~mm})$. The laser

174 beam diameter at the probed volume has been estimated to be $500 \mu \mathrm{m}$. The output laser fluence

175 was adjusted with an attenuator for each excitation wavelength, in order to maintain its value 176 below the saturation threshold and to avoid interference with the LII signal. Typically, the 177 measurements have been performed for fluence below $20 \mathrm{~mJ} \mathrm{~cm}^{-2}$. The absence of LII signal 178 has been systematically checked for each wavelength in the sooting region of the flame around $17980 \mathrm{~mm}$ above the burner.

180 The emitted fluorescence was collected at a right angle with two spherical lenses 181 (diameter $=50 \mathrm{~mm}$, and focal length $=100$ and $75 \mathrm{~mm}$, respectively) and imaged into a bundle 182 of optical fibers (diameter $=1.25 \mathrm{~mm}$ ) connected to a spectrometer (IHR320, Jobin Yvon). The 183 spectrometer was equipped with a 100 grooves $/ \mathrm{mm}$ grating blazed at $450 \mathrm{~nm}$. The fluorescence 184 spectra have been recorded using a 16 bit ICCD camera (PI-MAX 2, Roper Scientific) coupled 
to the spectrometer. The gatewidth $(\mathrm{GW})$ of the intensifier was fixed at $40 \mathrm{~ns}$. Prompt and

186 delayed fluorescence measurements after the laser pulse have been performed as discussed 187 below to discriminate the fluorescence signals issued from the PAHs and dimers of PAHs. The 188 spectral resolution of our detection system was $0.7 \mathrm{~nm} /$ pixel. The LIF decay signal at specific 189 wavelengths was also recorded with a photomultiplier tube (XP2020Q, Photonics). The 190 scattered laser light was removed by a WG 295 filter for laser wavelengths lower than $300 \mathrm{~nm}$ 191 or by notch filters for laser wavelengths higher than $400 \mathrm{~nm}(405,488$ and $532 \mathrm{~nm})$. The spectrometer has been wavelength-calibrated using the spectral lines from a mercury pen lamp. The fluorescence spectra were corrected for the spectral intensity response of the detection

194 system (lenses, fiber, grating and camera) by the means of a uniform light source (CSTM-LR195 6-M, SphereOptics). Laser induced incandescence (LII) experiments were performed at $1064 \mathrm{~nm}$ using the fundamental of a pulsed Nd:YAG laser (Quantel Brilliant B, $6 \mathrm{~ns}$ pulse width, $10 \mathrm{~Hz}$ ), already

198 reported in a previous paper ${ }^{33}$. Soot particles were heated below the sublimation threshold 199 with a top hat profile at a fluence of $130 \mathrm{~mJ} \mathrm{~cm}^{-2}$ and a square shape of the laser beam profile 200 (1x1,1 mm). The LII emission was recorded at $90^{\circ}$ incidence with respect to the laser beam 201 propagation, using a system of two achromatic lenses for the imaging of the selected volume 202 at the entrance of a spectrometer equipped with a 150 groves $\mathrm{mm}^{-1}$ grating blazed at $300 \mathrm{~nm}$. 203 A fast ICCD camera (Princeton Instruments PIMAX) placed at the exit of the spectrometer 204 recorded an image corresponding to 500 laser shots. The LII detection was performed during $20550 \mathrm{~ns}$ GW after the peak of the laser pulse. In this case, the LII emission spectra were 206 recorded in the 580-800 $\mathrm{nm}$ wavelength range, with a filter that cut all the emission below $207500 \mathrm{~nm}$. This way the contribution of the second emission order of the grating in the visible 208 recorded domain was minimized. The spectral response of the detection system was calibrated 
in intensity and wavelength using a similar procedure to the LIF measurements. The LII

210 signal was integrated over $5 \mathrm{~nm}$ wavelength range centered at $750 \mathrm{~nm}$.

\section{Results and discussion}

\subsection{Experimental spectra}

Experimental fluorescence spectra measured with UV and visible excitation at different

HAB are shown in fig. $\mathbf{2}$ and fig. $\mathbf{3}$, respectively. Spectra in fig. $\mathbf{2 a}$ show the raw intensity to highlight the predominance of the visible emission between 400 and $600 \mathrm{~nm}$ above $40 \mathrm{~mm}$ HAB. The contribution of UV emission between 300 and $400 \mathrm{~nm}$ below $40 \mathrm{~mm}$ HAB is better highlighted in fig. $\mathbf{2 b}$, in which all the spectra have been normalized to unit maximum. Hence, progressively less dominant as the visible emission rises at higher $\mathrm{HAB}$, leading to a global broadening and shift of the fluorescence to higher wavelengths. The fluorescence spectra obtained at the excitation wavelength $405 \mathrm{~nm}, 488 \mathrm{~nm}$ and $532 \mathrm{~nm}$ are reported in fig. 3. The fluorescence spectra recorded at $405 \mathrm{~nm}$ excitation wavelength correspond to a particular the next section. The two other sets of spectra recorded at $488 \mathrm{~nm}$ and $532 \mathrm{~nm}$ excitation 228 wavelength do not show any spectral evolution against HAB in strong contrast with the spectra recorded with UV excitation (fig. 2). The spectral structure measured at $488 \mathrm{~nm}$ excitation wavelength shows a maximum intensity centered around $510 \mathrm{~nm}$, while the

231 maximum intensity of the spectra obtained at $532 \mathrm{~nm}$ excitation is slightly red-shifted at 560 $232 \mathrm{~nm}$. 
In the literature, the analysis of fluorescence spectra obtained with UV excitation in 236 sooting flames is generally carried out by attributing different spectral ranges of the measured spectra to the emission of different classes of PAHs ${ }^{30,35}$. The following classification is commonly adopted: 2-3 rings PAHs are considered to fluoresce at 320-380 nm, while PAHs with 4 and more rings are expected to fluoresce above $400 \mathrm{~nm}$. Although this approach 240 provides an approximated discrimination of different classes of PAHs according to the size of 241 their aromatic system, it only enables a partial interpretation of the fluorescence spectra, 242 which is essentially limited by the possibility of overlap of different PAHs spectral contributions. To address this issue and get a more accurate interpretation of the fluorescence spectra, an original approach is hereby proposed that relies on the simulation of the fluorescence spectra with a database containing fluorescence spectra of PAHs and dimers of PAHs issued from the literature

\subsubsection{Spectroscopy-based arguments supporting PAH dimerization in flames}

The spectroscopy of PAH monomers is dominated by $\pi^{*}-\pi$ transitions, characterized by intense absorption bands in the UV region (200-300 nm), which shift and broadens toward longer wavelengths as the size of aromatic system increases. The corresponding fluorescence emission spectra of these species are characterized by emission bands in the spectral range 300-450 $\mathrm{nm}$. Shifting the excitation wavelength to the visible region therefore provides an

254 efficient way to selectively excite the largest PAHs. However, only a very limited number of 255 PAHs expected to be formed in flames are able to absorb above $400 \mathrm{~nm}{ }^{29,36,37}$.According to these last references, no PAH up to coronene possess strong enough absorption bands above $450 \mathrm{~nm}$, which could explain the intense fluorescence emission in the visible region measured in sooting flames at visible wavelength excitation. Hence, PAH monomers cannot be 
488 or $532 \mathrm{~nm}$ excitation wavelength. Moreover, usual PAHs spectral properties are not 261 consistent with the wavelength range $(450-700 \mathrm{~nm})$ of the fluorescence emission signal measured for high HAB in the flame. By contrast, dimers of moderate-sized PAHs, studied in liquid phase have been shown to possess strong absorption features in this visible region ${ }^{38}$. Although the spectroscopic schemes for such excitation wavelengths are not yet fully understood, clear experimental evidences have been reported ${ }^{38}$, notably for the dimers of pyrene and perylene, that highlight intense fluorescence emission following excitation at 488 $\mathrm{nm}$ and $532 \mathrm{~nm}$. The corresponding fluorescence emission spectra are characterized by Stokes and anti-Stokes spectral features around the excitation wavelength, very similar to the spectra measured in sooting flames.

\subsubsection{LIF spectral modeling}

In order to interpret the experimental emission fluorescence spectra measured in the flame, we have developed a spectroscopic model capable to simulate these spectra. This simulation code relies on a database containing the excitation and emission fluorescence spectra of twenty pericondensed PAHs, from naphthalene $\left(\mathrm{C}_{10} \mathrm{H}_{8}\right)$ to coronene $\left(\mathrm{C}_{24} \mathrm{H}_{12}\right)$, listed in table 1. We chose these PAHs as they represent the most stable and preferentially formed PAHs in sooting flames according to the HACA mechanism that is the preferential chemical

278 route for the PAHs growth in sooting flames ${ }^{39}$. In the database, we did not include spectra of PAHs larger than coronene, as such large PAHs are expected to form in very small quantities, 280 not consistent with the intense LIF signals observed in sooting flames.

281 We also considered the contribution of dimers of PAHs expected to form in flames in 282 sufficient concentration, i.e, dimers of moderate-sized PAHs also listed in table 1. Hence, five 283 homodimers of PAHs, from anthracene to benzo(ghi)perylene, have been taken into account. 284 It is noteworthy that, although heterodimers may also form in flames ${ }^{40}$, the lack of 
spectroscopic data effectively prevents their use in this work. LIF spectra are modeled 286 following the same procedure as used in Mouton et al. ${ }^{41}$ for the simulation of LIF spectra at 287 low temperature. The global simulated LIF spectrum corresponds to the weighted sum of the contributions of each individual species (PAHs and dimers of PAHs) according to this formula:

$$
\text { Calculated Spectrum } \sim I_{\text {laser }} \cdot \sum_{i}\left(\theta_{i} \cdot \sigma_{i}(\lambda) \cdot N_{i} \cdot \operatorname{Spectrum}\left(P A H_{i}, \operatorname{Dimer}_{i}\right)\right)
$$
absorption cross section at the excitation wavelength $\lambda, N_{i}$ the weight of the species (mainly proportional to their concentrations $)$ and $\operatorname{Spectrum}\left(P_{A} A H_{i}, \operatorname{Dimers}_{i}\right)$ the normalized emission fluorescence spectrum of each considered PAH or dimer.

The contributions of the different PAHs and dimers are determined by adjusting their relative concentration $N_{i}$ until the best agreement is found between the experimental and the global simulated spectrum. The fluorescence quantum yield $\theta_{i}$ has been considered constant for all species because of the lack of knowledge concerning this data.

The reference papers from which the absorption and emission spectra of PAHs and dimers of PAHs constituted the database are taken are reported in table 2 . In order to be consistent in terms of intensity and absorption lines positions, we only considered the excitation spectra measured in solution by Karcher et al. ${ }^{29,37}$ to build the database of our model. It would have been obviously more accurate to use PAH excitation spectra measured at high temperature in the gas phase, but these data were only available for naphthalene ${ }^{42}$, pyrene $^{43}$ and anthracene ${ }^{43}$ among the twenty PAHs included in our model. Hence, for the 306 sake of consistency, the use of these spectra would have required some corrections in 307 intensity to be comparable with the spectra measured in solution. 
concerning the main PAHs in our model up to benzo(a)pyrene. As the fluorescence emission

311 spectra we used in our code required to be normalized in intensity, we chose to include in the database PAH emission spectra measured at high temperature when they are available. database. The spectroscopy of these species relies on the formation of PAH excimers from the ground state dimers by the excitation of one of the two monomers (M---M) into a first local excitation (LE) state quickly followed by the stabilization of the excimer predominantly by exciton resonance $\left(\mathrm{M}^{*} \mathrm{M} \leftrightarrow \mathrm{MM}^{*}\right)$ and/or by charge resonance interaction $\left(\mathrm{M}^{+} \mathrm{M}^{-} \leftrightarrow \mathrm{M}^{-} \mathrm{M}^{+}\right)$ $25,44,45$. Note that excitons and charge resonance interactions are responsible for the preferred parallel arrangement of PAH excimers into stacked clusters that provide the smallest distance between the molecular centers. The molecular rearrangement between the two minima of the higher potential energy surfaces is shown in fig.4. The fluorescence emission of the excimer is directly issued from this state and gives rise to the redshifted (in comparison with the excitation wavelength), broad and unstructured fluorescence spectrum represented on the left side of fig.4.

As shown in this figure, the excimer fluorescence depends on the binding energy $\left(D_{e}\right)$ as well as the repulsive energy of the ground state (depending on the intermolecular distance). The maximum of the excimer fluorescence occurs from the bottom of the potential well of the $(\mathrm{M}-\mathrm{M})^{*}$ excimer to the ground state of the M---M dimer, whereas the emission at the smallest and highest wavelength comes from the outer edges of the well. Since the repulsive potential 330 of PAH dimers remain poorly understood, we based our simulations on the following consideration. We assumed that the binding energy can be defined as the difference between 332 the monomer and excimer fluorescence energy ${ }^{23}$. Hence, the energy bandwidth of the 333 excimer corresponds to the half width of the binding energy. As mentioned by Sumi ${ }^{46}$, a

334 fluorescence spectrum of an excimer in the liquid phase is a "broad Gaussian-like band". A 
similar shape is also observed in gas phase ${ }^{24}$. Consequently, excimer fluorescence spectra

336 have been fitted by a Gaussian function centered at the excimer energy provided by the 337 literature and characterized by a full width at half maximum (FWHM) equal to half of the 338 binding energy $D_{e}$. Fluorescence and binding energy of excimers are given in table 3 . It is 339 worth noting that regardless the reference, the binding and excimer fluorescence energy are 340 relatively close to each other. For this reason, we used an averaged value for each excimer. It is known from the literature that the excitation spectra of aromatic dimers resembles 342 monomers spectra ${ }^{25,47}$. They are only slightly shifted and broadened toward higher 343 wavelengths relative to the monomer absorption spectrum and have a longwave "trail" 47 . For 344 this reason, we considered in our model, for the UV excitation wavelengths, that the molar 345 extinction coefficients of the monomer were representative of the molar excitation 346 coefficients of the dimers. Concerning the visible excitation of the dimers, there is only very 347 little information available in the literature. However, it has been clearly highlighted that 348 dimers of PAHs as pyrene and perylene, which are both used in our simulation code, can 349 absorb at 488 and $532 \mathrm{~nm}$ and give rise to broad band emission fluorescence spectra in the 350 visible spectral region ${ }^{38}$. We considered an equal probability for their excitation in the visible 351 range $(405,488$ and $532 \mathrm{~nm})$ for the five selected dimers. The intensities of the fluorescence emission spectra were therefore only weighted by the adjusted value of the respective relative concentrations $N_{i}$ of each dimer.

From a numerical analysis point of view, at present we cannot rule out the existence of 355 multiple solutions of equation 1. Finding all the possible solutions that minimize the distance 356 between the measured fluorescence spectrum and the linear combination of PAHs/dimers 357 spectra within a certain residual is equivalent to estimating the number of local minima of an 358 unknown n-dimensional surface. In order to obtain a deep and reliable understanding of the 359 fluorescence emission in the visible, this is an important albeit not trivial challenge that will 
360 be tackled in the future but currently goes beyond the scope of this paper. However, the

361 existence of multiple solutions does not affect the main conclusion of our investigation: there

362 are no linear combinations of the fluorescence spectra of twenty selected PAHs assumed to be

363 important soot precursors in the literature that can reproduce the experimentally observed

364 fluorescence emission in the visible. In turn, a handful of the fluorescence spectra of PAH

365 dimers are enough to result in (at least) one linear combination that very closely reproduces

366 the aforementioned fluorescence emission in the visible.

\subsection{Analysis of the fluorescence emission spectra}

Experimental and calculated spectra are reported in fig. $\mathbf{5}$ and fig. 6 . The red solid line

represents the experimental spectra, while the blue solid line corresponds to the global

calculated spectrum, sum of the individual contributions of PAHs (thin colored solid lines) and dimers (thin colored dashed lines).

\subsubsection{Fluorescence induced by 213.5 and $282 \mathrm{~nm}$ excitation wavelengths}

At both UV excitation wavelengths, the excellent match of experimental and calculated spectra shows that the UV fluorescence emission $(300-450 \mathrm{~nm})$ comes from the

376 fluorescence of PAH monomers. This spectral feature is mainly constituted of the spectral contributions of naphthalene, pyrene and fluoranthene, which all possess strong oscillator strengths at both excitation wavelengths. The contributions of fluorene, anthracene,

379 phenanthrene are also noticeable below $20 \mathrm{~mm} \mathrm{HAB}$, while the contributions of

380 benzo(a)pyrene, perylene and benzo(ghi)perylene appear higher in the flame. It is worth 381 noting that only nine over the twenty available PAHs in our database are necessary to correctly reproduce the UV part of the fluorescence spectra, and this further strengthens the 383 observation that multiple linear combinations of PAHs spectra may lead to simulated spectra 384 very similar to the experimental spectrum. 
Above $15 \mathrm{~mm} \mathrm{HAB}$, we observe the emergence of a contribution in the visible region beyond $500 \mathrm{~nm}$ that cannot be reproduced by any combination of the PAH monomers spectra in our database. To correctly reproduce the entire experimental spectra, an additional contribution coming from three dimers of PAHs (pyrene, benzo(a)pyrene, perylene) is required (fig. 5). This addition leads to an excellent agreement with the experimental spectra all along the flame height, showing that the visible part of the emission spectra can only derive from dimers of moderate-sized PAHs and not from large PAHs. This clearly means that the common interpretation correlating the visible emission of such spectra measured in flames to the fluorescence of different classes of large PAHs is not adequate and leads to flawed conclusions. Moreover, we show here that, with the only exception of naphthalene, the strong overlap of the different spectral contributions of the excited PAHs prevents any precise distinction of classes of PAHs based on the fluorescence wavelength range.

\subsubsection{Fluorescence induced by $405 \mathrm{~nm}$ excitation wavelength}

Only three PAHs (fluoranthene, benzo(a)pyrene and perylene) out of the twenty in our database possess strong enough absorption bands to be excited at $405 \mathrm{~nm}$. Fluoranthene spectroscopy features an anomalous broadband excitation spectrum ${ }^{48}$ strongly red-shifted in comparison to PAHs of the same size (peak emission at $450 \mathrm{~nm}$ against $390 \mathrm{~nm}$ for pyrene). The experimental and simulated spectra obtained at $405 \mathrm{~nm}$ excitation wavelength are shown in the left column of fig. $\mathbf{6}$. The spectral analysis reveals that the structure of the experimental spectra is well reproduced by the contributions of fluoranthene, benzo(a)pyrene and perylene at low $\mathrm{HAB}$, while supplementary contributions of dimers of pyrene, benzo(a)pyrene and perylene are required higher in the flame. The increase of the excitation wavelength from the UV range to $405 \mathrm{~nm}$ clearly limits the pool of excitable PAHs and constitutes an experimental approach to selectively excite the largest PAHs formed in the flame. Moreover, it implicitly 
underlines the formation of dimers of PAHs which, as for UV excitation, strongly contributes

411 to the visible part of the measured fluorescence emission spectra.

\subsubsection{Fluorescence induced by 488 and $532 \mathrm{~nm}$ excitation wavelengths}

The fluorescence spectra shown in the last two columns in fig. 6 are obtained with 415 visible excitations (488 and $532 \mathrm{~nm}$ wavelength, respectively). As shown in the figure, there 416 is no spectral evolution of the global emission structure against HAB in strong contrast with 417 the spectra recorded with UV excitations. Here, the spectra feature intense Stokes and anti418 Stokes components from both sides of the excitation wavelength. No PAHs can be excited 419 anymore at these wavelengths and the simulated spectra involve only dimers of PAHs. In 420 particular, an excellent agreement between the simulations and the experimental spectra 421 acquired at $488 \mathrm{~nm}$ excitation wavelength is obtained at any HAB with the dimer of benzo(a)pyrene and a smaller contribution from the dimer of pyrene and perylene. The simulation of the spectral structure measured at $532 \mathrm{~nm}$, requiring the contribution benzo(a)pyrene and perylene, is also satisfying, except for the edges near $700 \mathrm{~nm}$. This discrepancy may be attributed to the limited number of dimers in our database. The overall fit highlights that the spectral shift of the maximum intensity of the spectra from 510 to $560 \mathrm{~nm}$, is due to a more efficient excitation of the dimer of perylene at $532 \mathrm{~nm}$ in comparison to the excitation at $488 \mathrm{~nm}$.

\subsection{Effect of the delay on the recorded LIF spectra}

Another strong argument for dimers optical detection in flames relies on the 432 fluorescence lifetimes of the excited species. It is known that dimers of PAHs have 433 substantially longer fluorescence lifetimes than PAH monomers 44,49 , which can be 434 highlighted by delaying the detection gate width of the fluorescence signals. In order to 
strengthen the evidence of dimer formation in the sooting flame, we carried out a series of

436 LIF measurements in which we varied the gate delay (GD) of the ICCD camera with respect 437 to the peak of the temporal LIF signal, considered to be the prompt fluorescence in this case. 438 All the delayed LIF spectra have been recorded with the same 40 ns gate width, but 439 temporally shifted in order to observe the species with longer $\tau$. Fig. 7 shows the LIF spectra 440 measured at $60 \mathrm{~mm} \mathrm{HAB}$ at $213.5 \mathrm{~nm}$ excitation wavelength and for different GD values. We have shown above that PAHs and dimers of PAHs can be excited at $213.5 \mathrm{~nm}$ and

442 fluoresce in two different spectral ranges. From our database, it appears clearly that PAHs 443 mainly fluoresce in the spectral range $300-450 \mathrm{~nm}$, while dimers of PAHs feature strong 444 fluorescence bands in the spectral range 400-700 nm. In fig. 7, we show that two different 445 spectral contributions can be distinguished according to the GD. The UV part of the spectrum, 446 characteristic of the PAHs fluorescence spectral range, is indeed strongly reduced with a late 447 detection while the LIF delayed detection temporally favors the red-shifted LIF signal coming 448 from the excited dimers. Note that the measured fluorescence spectra remain unchanged when 449 GD $>5 \mathrm{~ns}$, featuring a very short fluorescence decay time for the PAHs in flames as already 450 observed in the literature ${ }^{50}$. We also performed the same experiments with a visible 451 excitation wavelength at $488 \mathrm{~nm}$. In this case, not reported here, we did not observe any 452 change in the shape of the LIF spectra regardless of the time delay. This observation clearly 453 denotes the excitation at visible wavelength of a same sort of species characterized by 454 fluorescence spectra pointing to the fluorescence of dimers of PAHs. As we have shown above, the contributions of PAHs and dimers of PAHs to the global 456 fluorescence spectrum evolve with the HAB. We also performed delayed experiments at 457 different HAB. In fig. 8, we report a comparison of three LIF spectra, measured at $213.5 \mathrm{~nm}$ 458 excitation wavelength with and without applying a delayed detection of $10 \mathrm{~ns}$ for three 459 different HAB. 
At $30 \mathrm{~mm} \mathrm{HAB}$, the important contribution to the UV region of the spectrum disappears by delaying the detection, confirming the simultaneous important presence of PAHs and dimers of PAHs in this zone of the flame. The impact of the delayed detection on the measured spectrum is slightly reduced at higher HAB due to the smaller contribution of the PAHs fluorescence to the global spectrum. However, this systematic depletion provides another indication that PAHs contribute to the fluorescence spectra in the spectral range 300$450 \mathrm{~nm}$ up to $60 \mathrm{~mm} \mathrm{HAB}$, consistent with the spectral analysis provided in the main text. $\mathrm{nm}$ and $532 \mathrm{~nm}$. In this case, no spectral change of the emission spectrum was observed at any $\mathrm{HAB}$. Here, this observation is characteristic of the only excitation of dimers of PAHs with visible laser wavelengths.

As expected from their spectroscopic properties ${ }^{38}$ and confirmed by this work, dimers of PAHs can be excited both in the UV and the visible range. This property can be highlighted by comparing the fluorescence spectra obtained for different excitation wavelengths. In fig.9, we show a comparison between two prompt fluorescence spectra recorded at 488 and $532 \mathrm{~nm}$ excitation wavelength and one fluorescence spectrum obtained at $213.5 \mathrm{~nm}$ excitation wavelength and temporally delayed to exclude PAHs fluorescence emission. From this figure, it appears that these spectra are characterized by a similar broadband and structureless spectral shape, only slightly red-shifted according the excitation wavelength from the UV to visible. The slight shift between the different spectra can be attributed, as highlighted with our model, 480 to the fluorescence of different dimers of PAHs, the intensities of which may be favored by 481 the choice of the excitation wavelength, or by changes of their emission properties with the 482 excitation wavelength as proposed elsewhere ${ }^{38}$. 
The detailed analysis of the fluorescence spectra of the sooting flame enables to 486 clearly attribute the measured intense visible LIF signal to the spectral signature of dimers of moderate-sized PAHs. Moreover, we show that the contributions of several dimers are necessary to properly reproduce the experimental spectra. Fig. 10 shows the relative evolution against the flame HAB of the normalized LIF signals of the main PAHs and dimers of PAHs, corresponding to the maximum LIF intensities determined for each species from the simulated spectra of fig. 5 and fig. 6. The dimer profile corresponds to an average profile of all the PAHs dimers as their shapes are very similar regardless of the dimers. The relative soot volume fraction profile (black line), previously measured ${ }^{33}$ by laser induced incandescence (LII), is also shown in fig. 10. These data highlight that the formation of the dimers starts slightly above the formation of PAHs (around $20 \mathrm{~mm}$ HAB) and reaches a maximum around $60 \mathrm{~mm} \mathrm{HAB}$, which corresponds to the beginning of the soot inception region. Consumption of dimers corresponds to the formation of soot particles, which ends when the pool of dimers has been fully consumed.

These results consequently support the hypothesis of kinetic rather than 500 thermodynamic control frequently used in emerging soot formation mechanisms ${ }^{15,19-21}$. For 501 instance Eaves et al. ${ }^{15,19}$ introduced in their models the concept of the reversibility of the 502 nucleation, implying that nucleation may not be governed by equilibrium processes as already suggested by the pioneering work of Dobbins et al. ${ }^{51}$. In this framework, the formation of dimers of moderate-sized PAHs is still a reversible process, however nucleation requires the dimers stabilization by the formation of a strong chemical bond as also proposed by Kholghy et al. ${ }^{21}$ through a sequence of hydrogen abstraction and carbon-carbon bond formation or dehydrogenation.

Another hypothesis to explain the stabilization of PAH dimers in flames proposes the 509 formation of aliphatic bridges between two PAHs. This suggestion is supported with 
510 experimental results in the recent work of Adamson et al. ${ }^{52}$, in which they detected and 511 identified aliphatically bridged PAHs in an ethylene/ $\mathrm{O}_{2}$ coflow diffusion flame with a tandem mass spectrometer. Although the geometrical arrangement of the two bridged PAHs could not

513 be determined in the work of Adamson et al. ${ }^{52}$ we believe that these species might as well 514 correspond to the same species fluorescing in the visible domain and observe by LIF in our 515 work. The PAHs connected by a short carbon chain form a stable intramolecular PAH dimer 516 involving the two bridged PAHs into a parallel arrangement involving van der Waals forces. 517 Under this configurations, such intramolecular dimers have been shown to provide similar 518 spectral signatures under laser excitation as intermolecular PAH dimers ${ }^{45}$ (dimers only 519 connected by van der Waals forces). Whether aliphatic bridged PAHs are formed in flames, 520 they probably rearrange themselves into intramolecular PAHs dimers, the dimerization 521 process being facilitated by the carbon chain connecting the two monomers. Alternatively, 522 intermolecular dimers of PAHs could also be first formed and stabilized by the formation of 523 an aliphatic chain instead of a simple carbon bond as envisaged by Kholghy et al. ${ }^{21}$, between 524 the two PAHs according to a similar irreversible process as proposed by this group ${ }^{21}$. It is clearly beyond the scope of this work to discuss the detailed kinetics leading to 526 the formation of these dimers, the main objective of this paper being to bring experimental 527 evidence supporting the formation of dimers of PAHs in a methane diffusion flame. However, 528 the formation of strong carbon bonds between two PAHs is likely a required step to stabilize 529 the dimer and initiate the process of nucleation. According to our experiments, we cannot 530 state if these chemical bonds are formed after the dimers as proposed by Eaves et al. ${ }^{19}$ or 531 Kholghy et al. ${ }^{21}$, or before according to the hypothesis of aliphatic bridges. However, all 532 these results clearly point to the dimers of moderate-sized PAHs being the elemental building 533 blocks in the soot nucleation process. 
In this work, we carried out the experimental study of a laminar diffusion sooting flame

of methane by laser induced fluorescence (LIF). A large database of emission fluorescence spectra has been recorded at different $\mathrm{HAB}$ along the vertical centerline of the flame and for different UV and visible excitation wavelengths. The analysis of these spectra has been

540 performed by the use of a simple model relying on a spectroscopic database containing the 541 excitation and emission fluorescence spectra of twenty pericondensed PAHs and five dimers of PAHs. From the analysis of the spectra provided by the comparison of the experimental and simulated spectra, we clearly distinguished two main contributions in the emission 544 spectra. The first contribution, dominant for the first HAB and ranging from 300 to $450 \mathrm{~nm}$, is 545 undoubtedly attributed to the fluorescence of some specific PAHs from naphthalene to 546 benzo(ghi)perylene. By contrast, we showed that the second contribution, occurring for higher $547 \mathrm{HAB}$ in the flame and characterized by an intense and unstructured emission spectra at 400$700 \mathrm{~nm}$, could not be reproduced by any combination of PAHs spectra. Based on 549 spectroscopic considerations, it turns out that this second contribution is very likely 550 characteristic of the emission of moderate-sized dimers of PAHs.

551 The analysis of the measured spectra clearly supports the formation of dimers of 552 moderate sized PAHs in the inception region of a sooting flame, very likely meaning that 553 these species play a significant role in the soot nucleation process. These results are consistent 554 with recent kinetic models that consider dimers as an intermediate species conditioning the 555 transition to soot particles ${ }^{19,21}$. It is currently not clear if dimers could be extracted from 556 flames with typical methods commonly used for flame investigations (as molecular beam 557 mass spectrometry for instance). Indeed, due to their brief lifetime and poor thermodynamic 558 stability, this certainly represents a challenging task and laser diagnostic might be the most 559 suitable choice to characterize these species. 
561 chemical bond between the two monomers, there might be some opportunities to extract these 562 species or to study them indirectly. In this case, it is probable that the parallel arrangement of 563 the dimer structure due to van der Waals forces could not be preserved and the sampled 564 species might therefore correspond to the species identified by Adamson et al. ${ }^{52}$. Surely, much work remains to be done to determine the exact nature of these species 566 and the mechanisms involved in the nucleation step. Nevertheless, the analysis we provided 567 sheds new light on the soot nucleation. The very existence of dimers of moderate-sized PAHs 568 in the nucleation region of sooting flames is strong evidence that soot nucleation is kinetically 569 rather than thermodynamically controlled, which is valuable information for the advancement 570 of modeling and the understanding of the soot formation process at its fundamental level.

\section{$571 \quad$ Conflicts of interest}

572 There are no conflicts of interest

\section{Acknowledgements:}

575 This work was supported by the Agence Nationale de la Recherche through the LABEX 576 CAPPA (ANR-11-LABX-0005), the Région Hauts-de-France, the Ministère de 577 l'Enseignement Supérieur et de la Recherche (CPER Climibio) and the European Fund for 578 Regional Economic Development. 


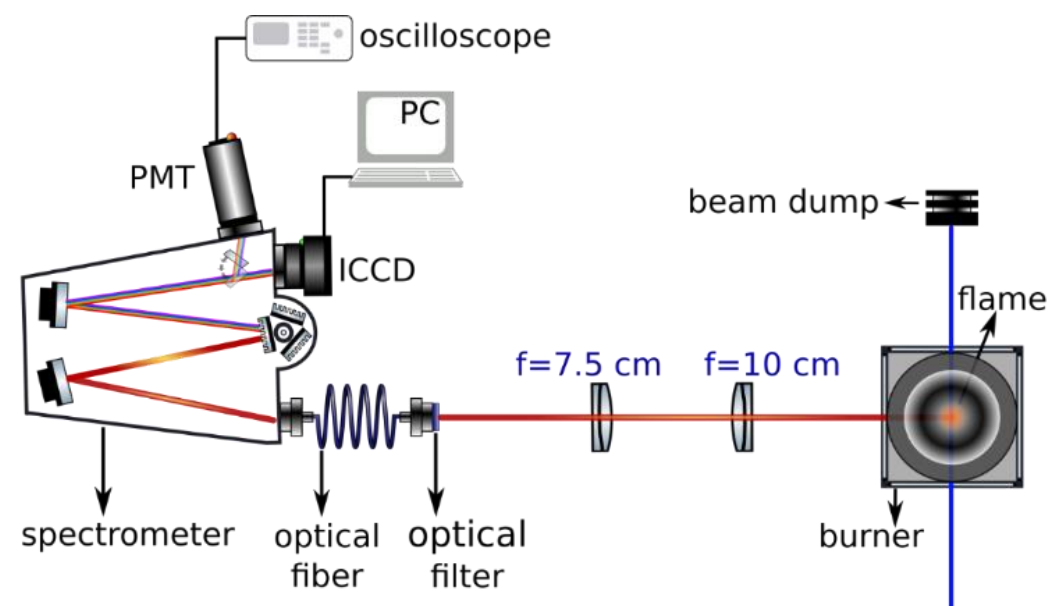

Figure 1: Experimental configuration for the LIF measurements.

584 

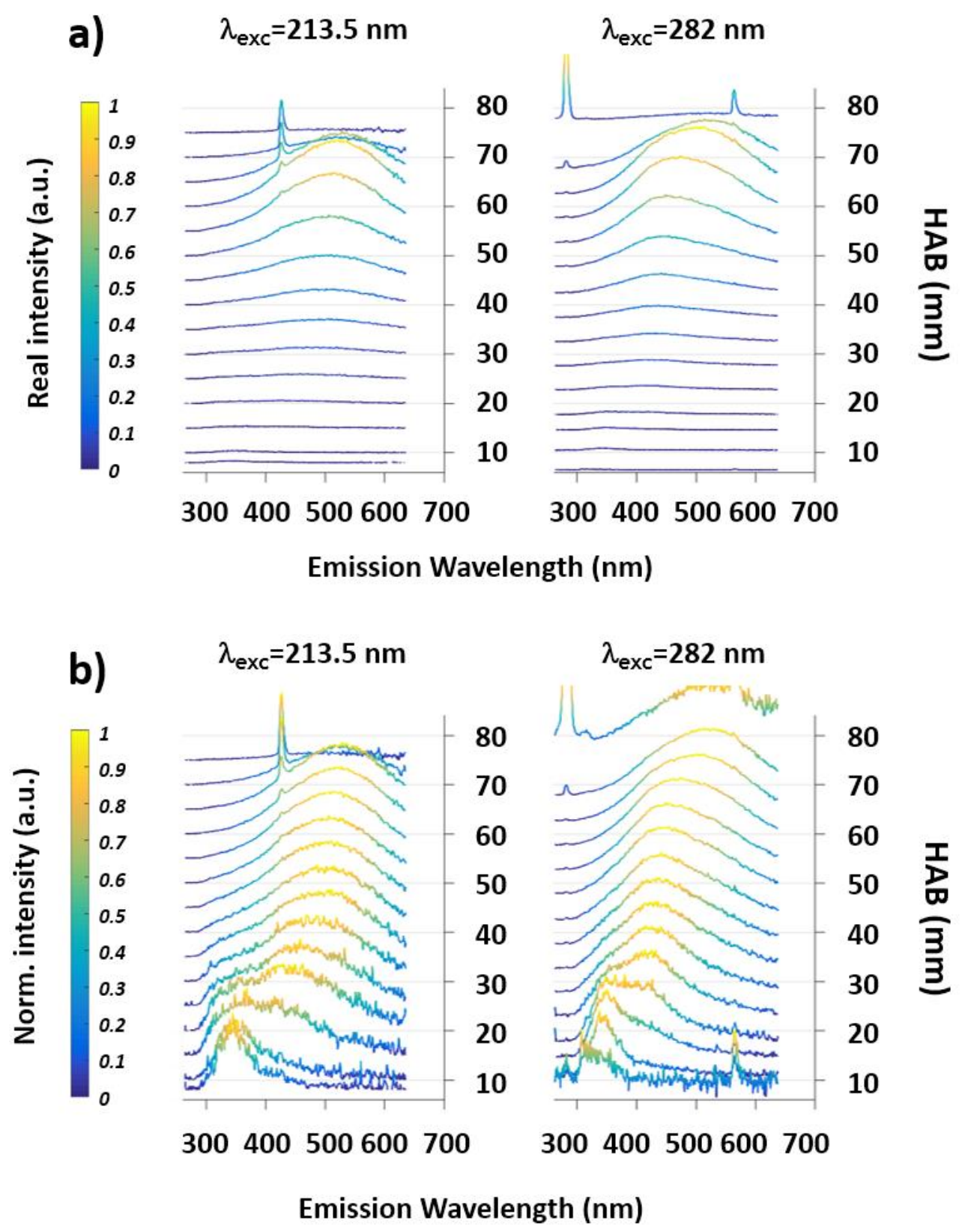

Figure 2: Fluorescence emission spectra recorded along the vertical centerline of the flame for two UV excitation wavelengths. a) Raw intensity (a.u.) b) Normalized intensity (a.u.) the laser excitation wavelengths. 


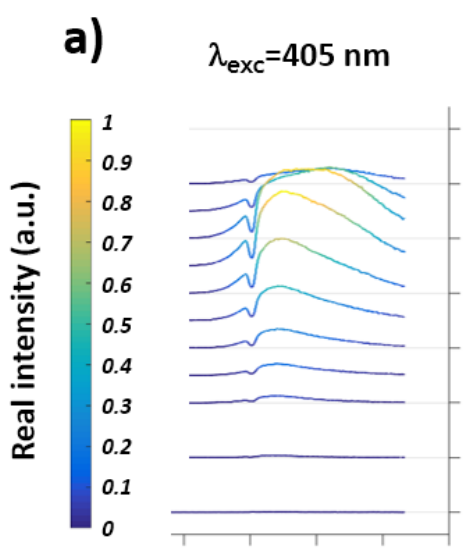

300400500600700

80
70
60
50
40
30
20
10

300400500600700

$\lambda_{\text {exc }}=488 \mathrm{~nm}$

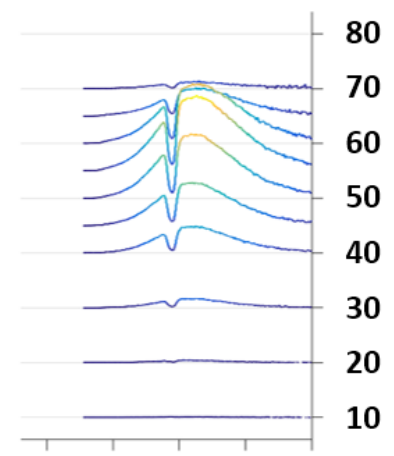

$\lambda_{\text {exc }}=532 \mathrm{~nm}$

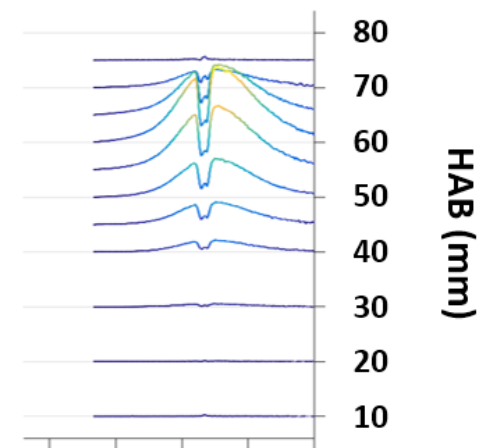

300400500600700

Emission Wavelength (nm)

b) $\quad \lambda_{\text {exc }}=405 \mathrm{~nm}$

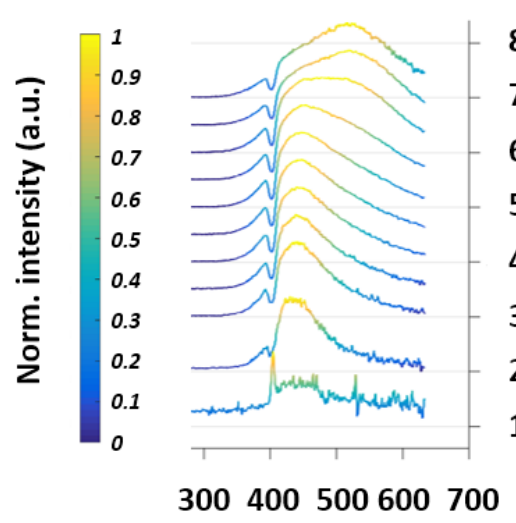

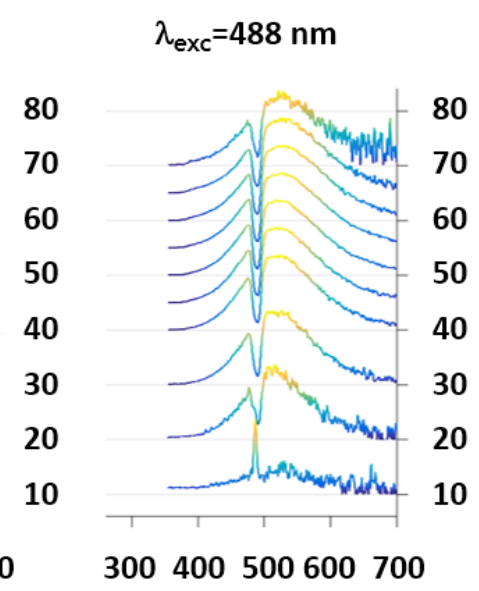

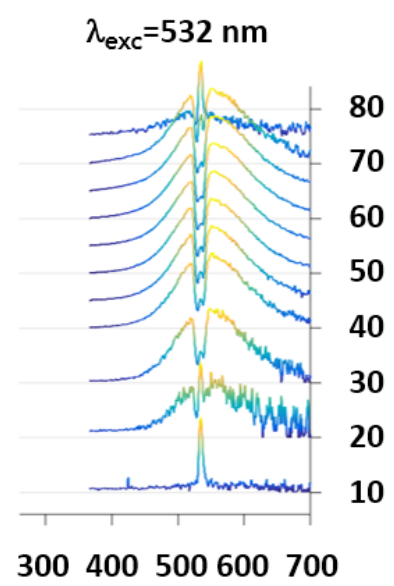

\section{$\frac{\substack{\mathbf{D} \\ \frac{\mathrm{m}}{3}}}{3}$}

Emission Wavelength (nm)

Figure 3: Fluorescence emission spectra recorded along the vertical centerline of the flame

594 for three visible excitation wavelengths. a) Raw intensity (a.u.) b) Normalized intensity (a.u.). The 


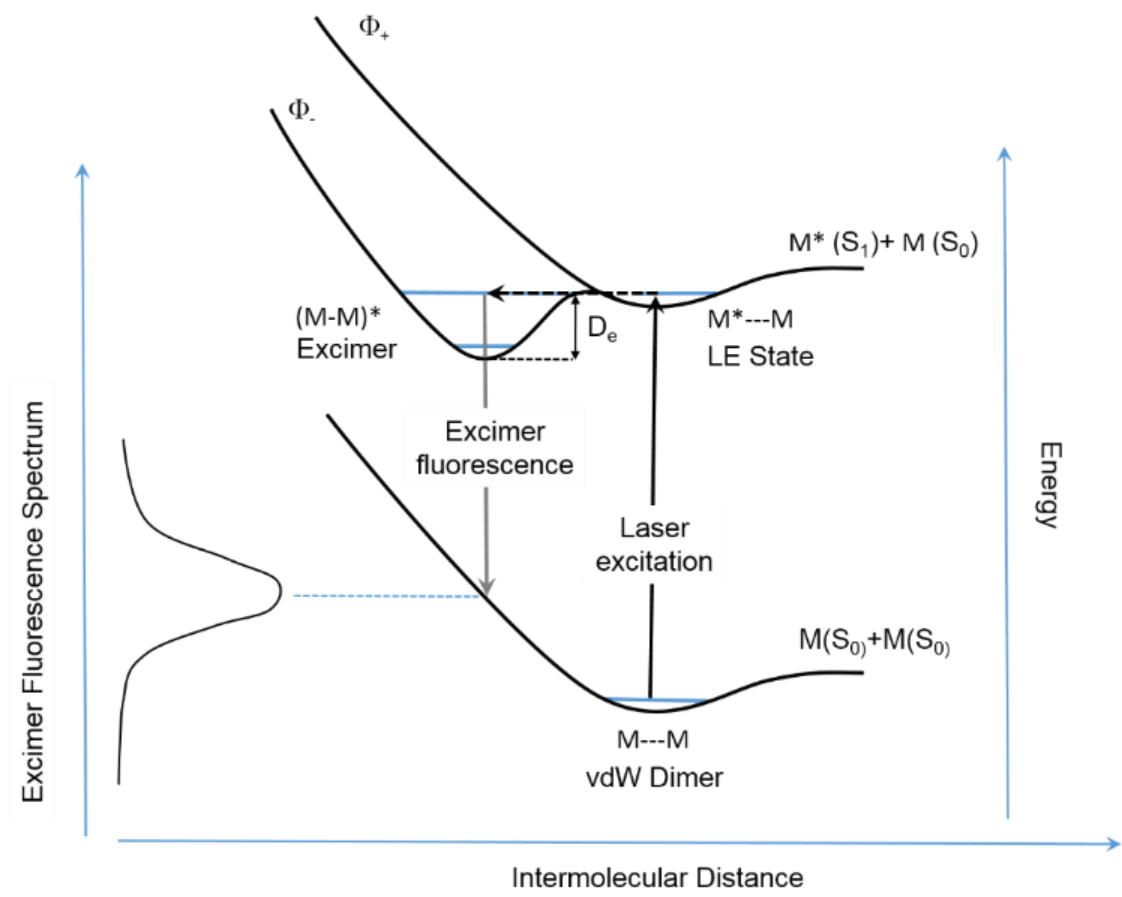

Figure 4: Schematic potential energy diagram for an excimer formation (M-M)* via the 


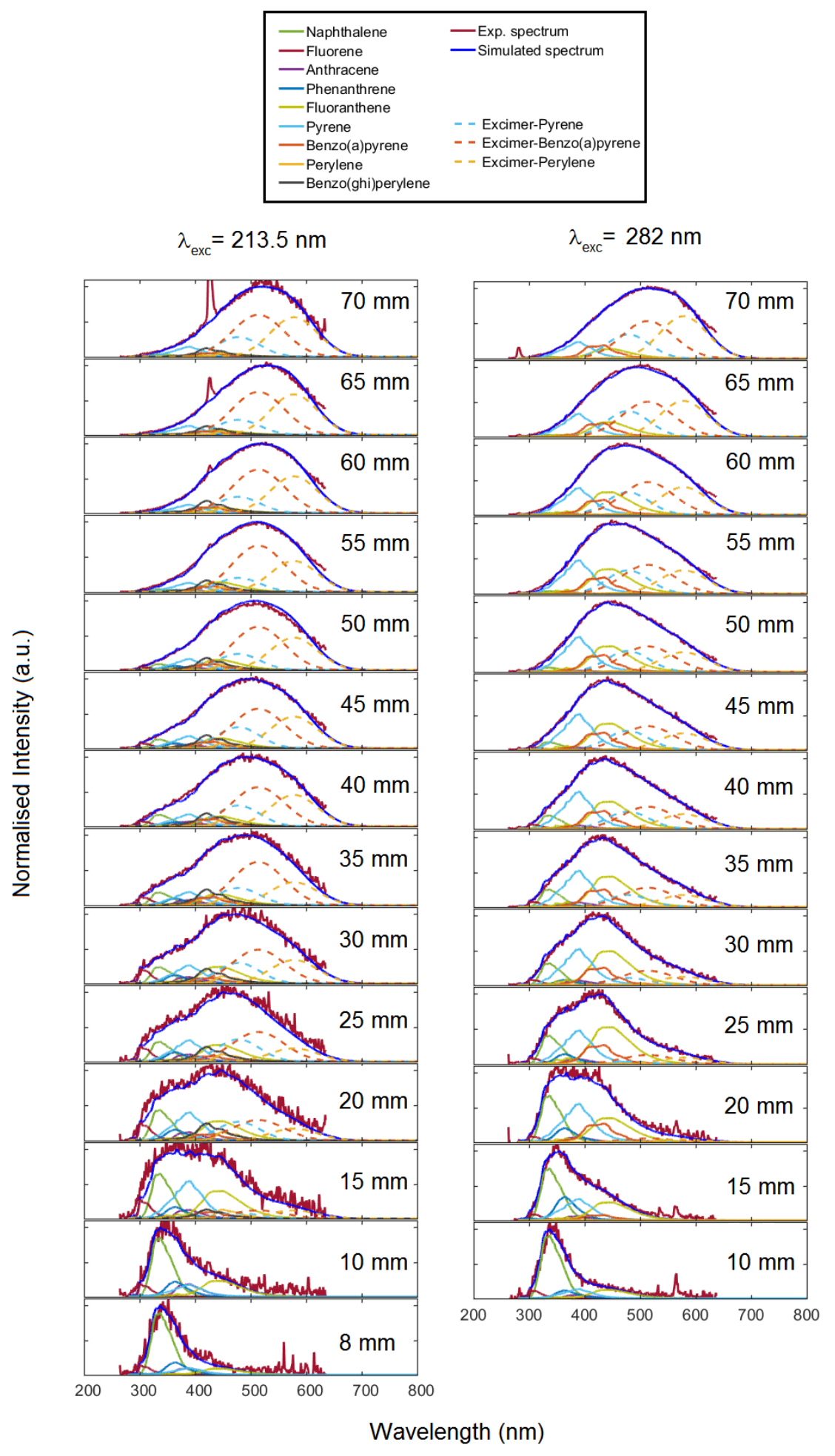

$608 \quad$ Figure 5: Calculated and experimental fluorescence emission spectra obtained for different UV

609 excitation wavelengths along the vertical centerline of the flame against HAB 

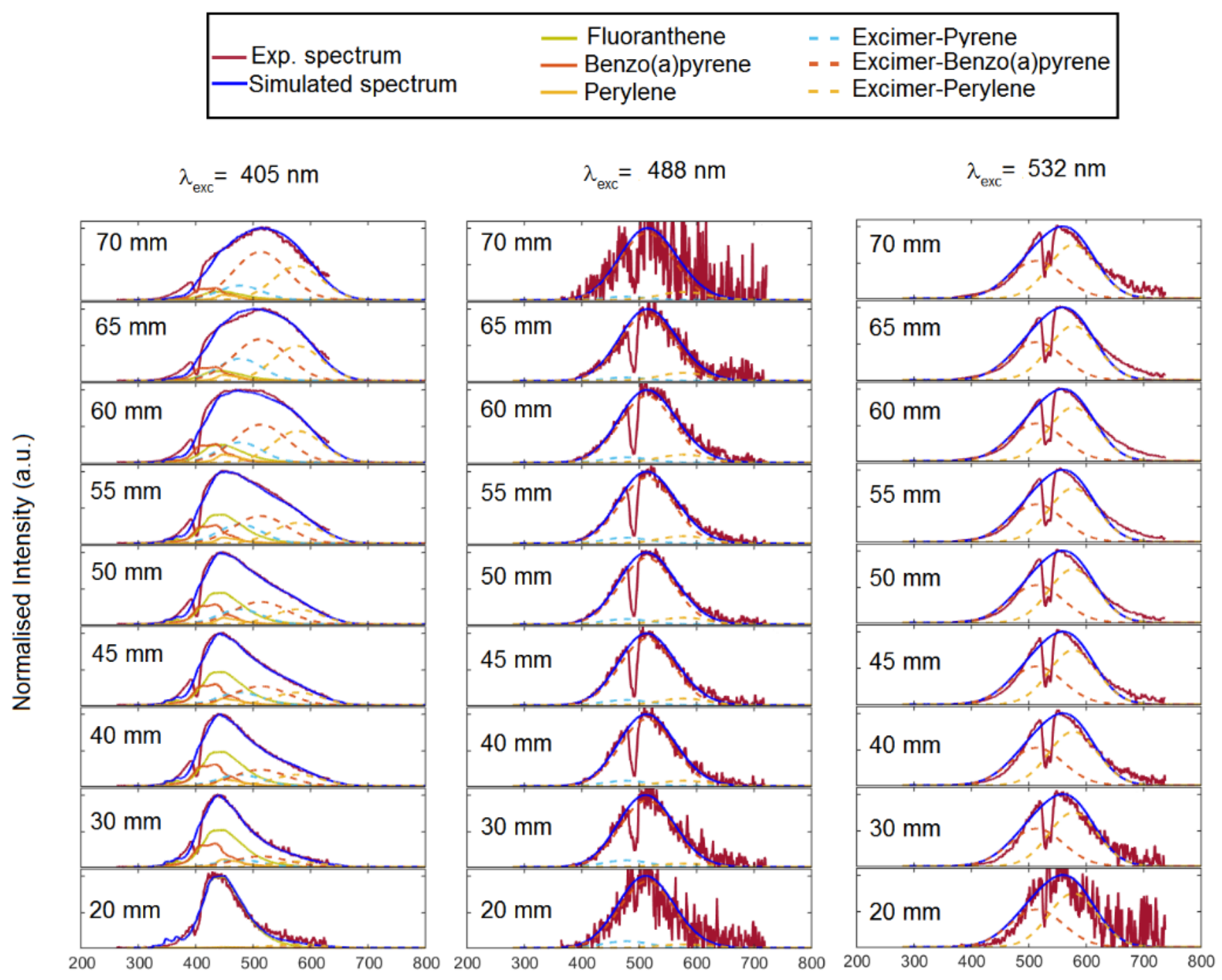

Wavelength $(\mathrm{nm})$

612 Figure 6: Calculated and experimental fluorescence emission spectra obtained for different visible excitation wavelengths along the vertical centerline of the flame against HAB.

614

615

616

617 


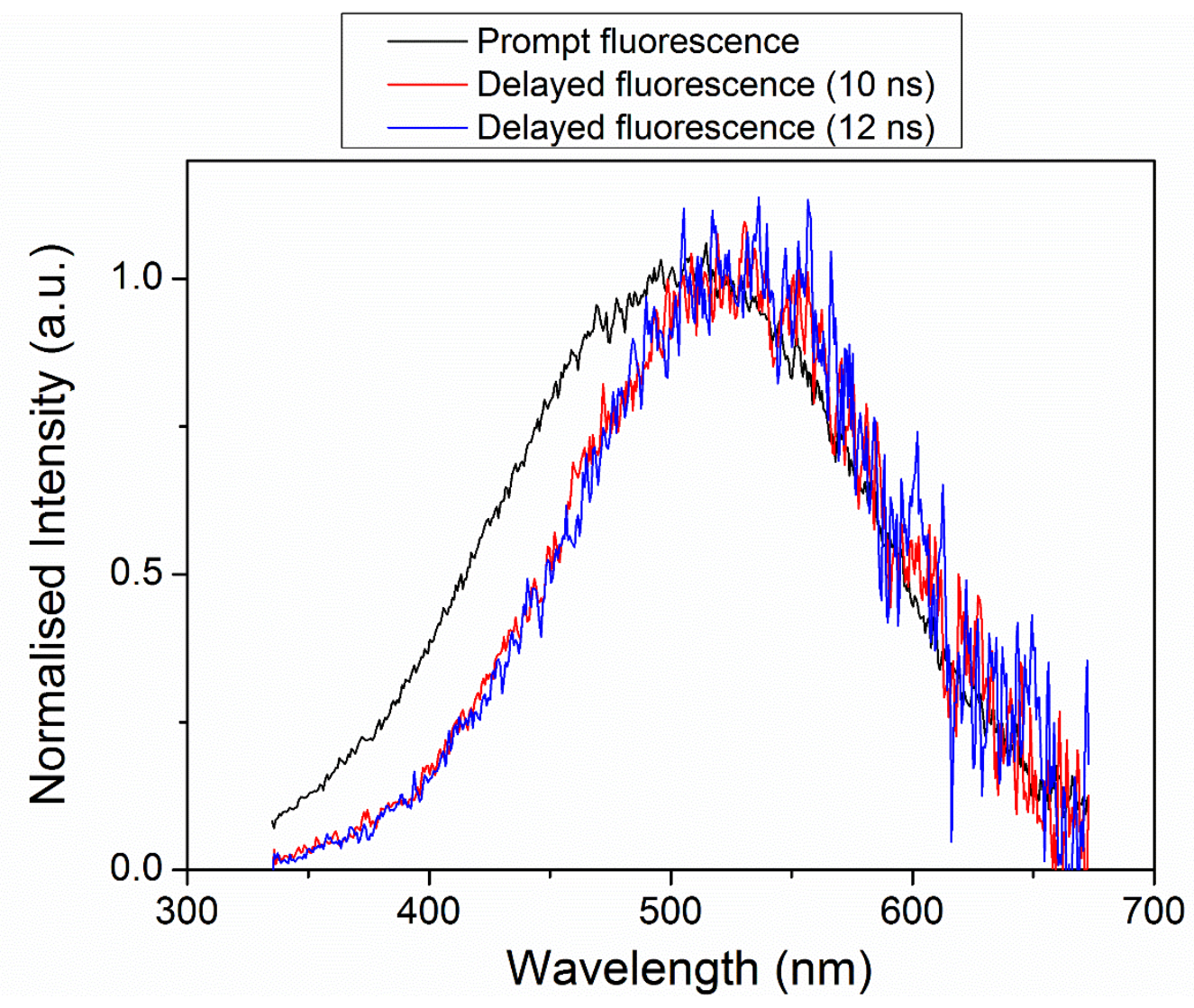

619

Figure 7: Prompt and delayed fluorescence spectra measured at $60 \mathrm{~mm} \mathrm{HAB}$, at $213.5 \mathrm{~nm}$ excitation wavelength. 


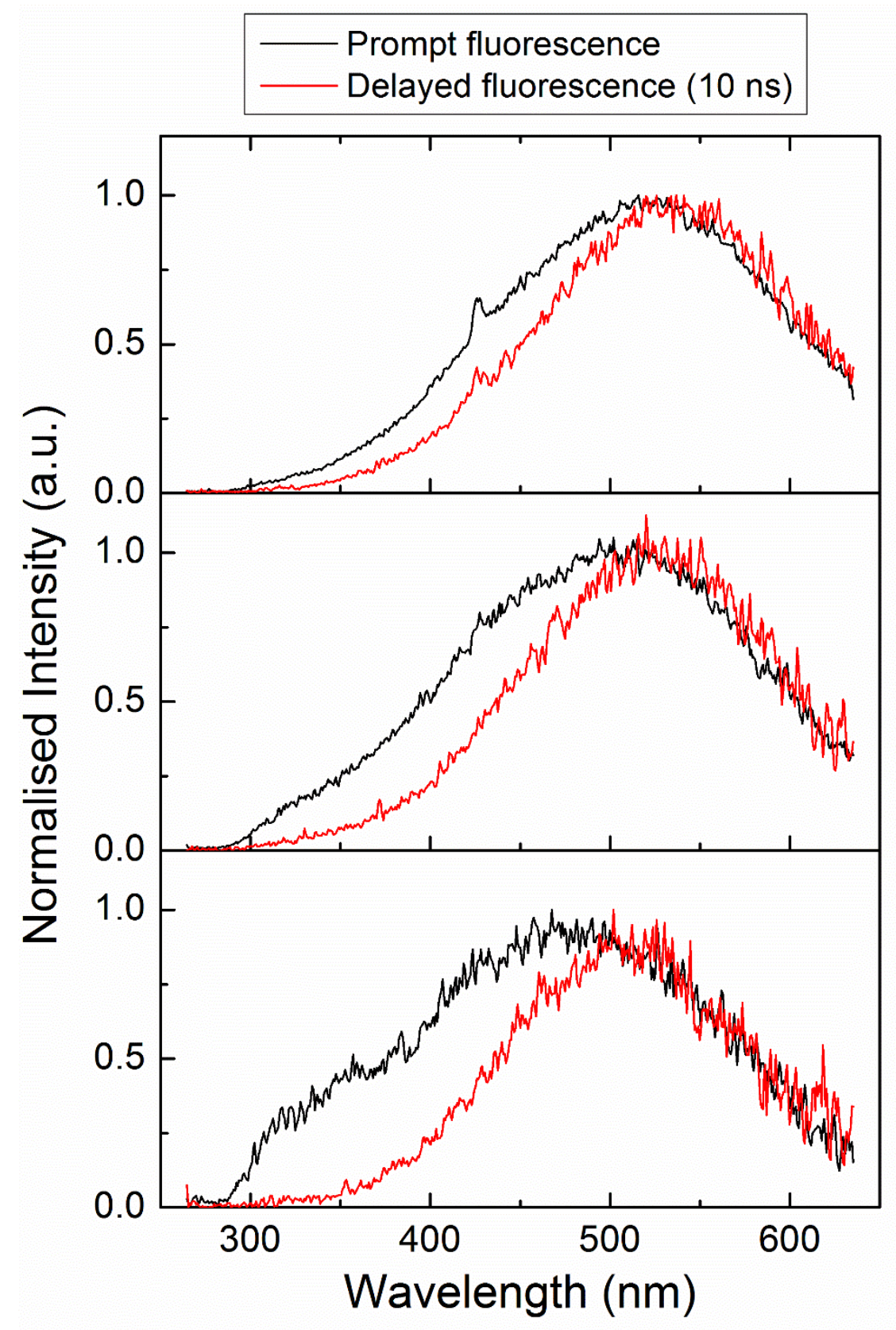

623

Figure 8: Comparison between prompt and delayed fluorescence spectra at $213.5 \mathrm{~nm}$ 


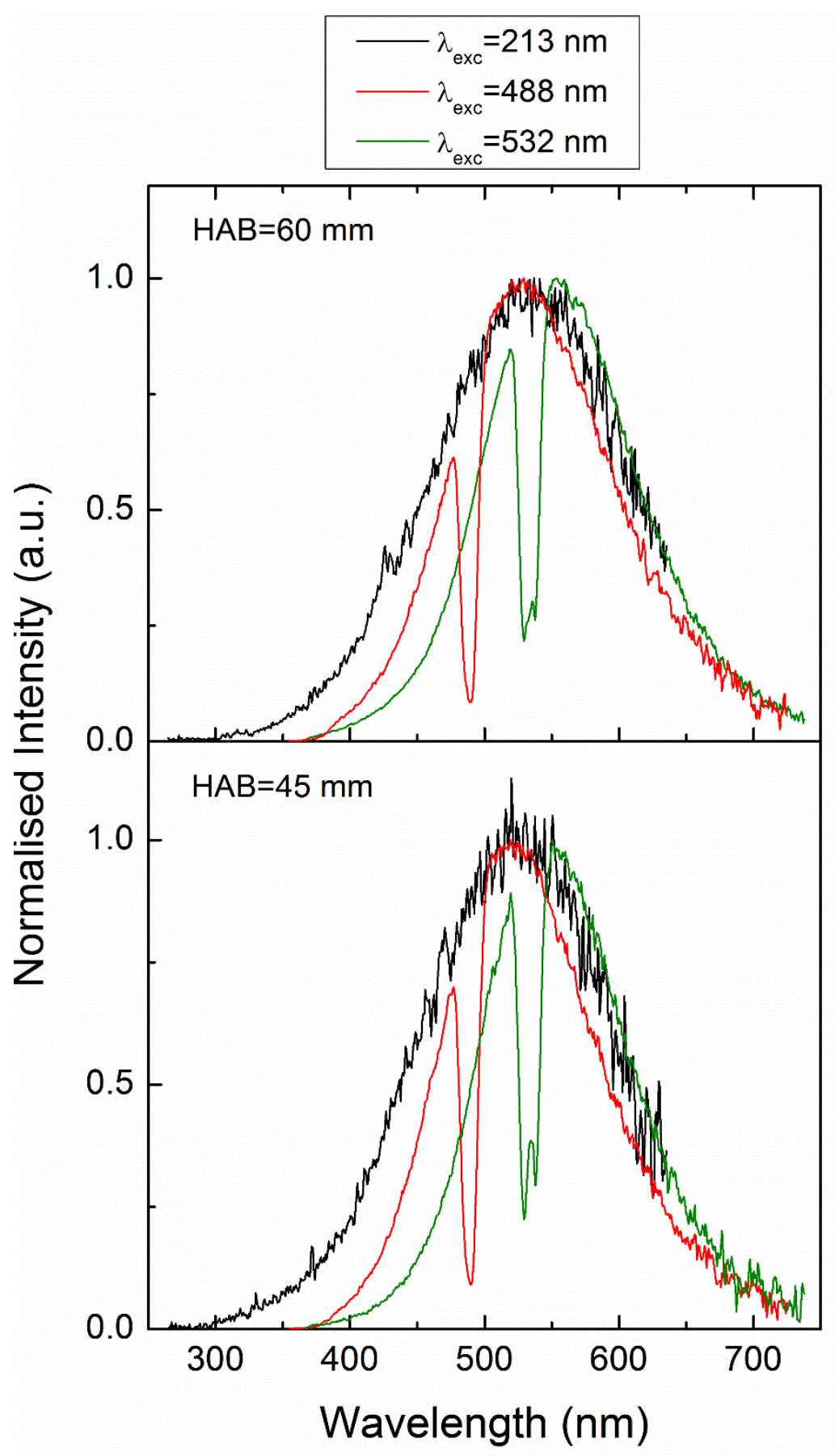

Figure 9: Comparison between delayed fluorescence spectra at 45 and $60 \mathrm{~mm}$ for 213.5, and 


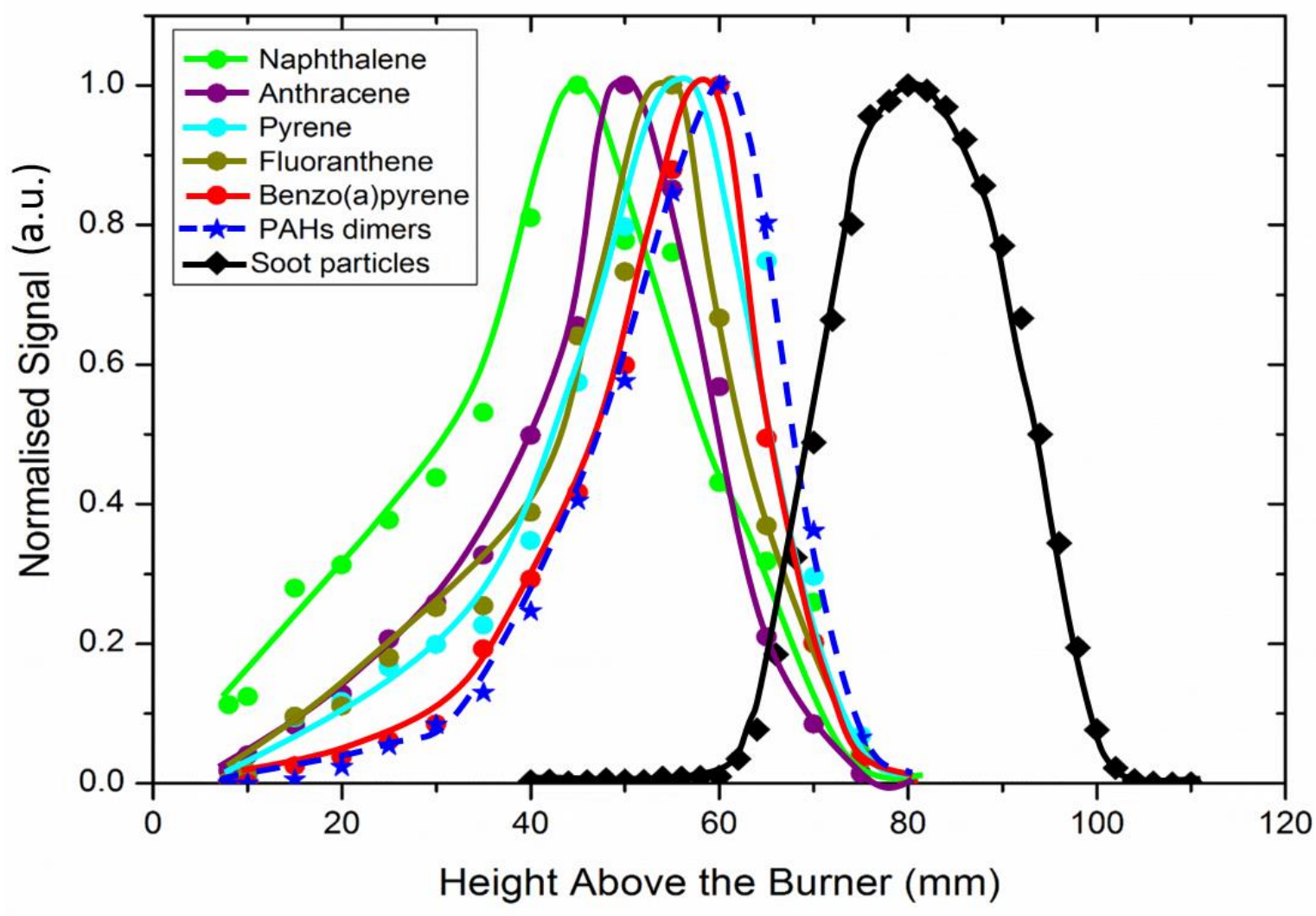

632

Figure 10: Specific profiles of PAHs and dimers subtracted from the LIF intensities determined from

634 the simulated spectra obtained along the centerline of the flame. The dimers profile is an average

635 profile of the dimers profile of pyrene, benzo(a)pyrene and perylene. The soot profile has been 


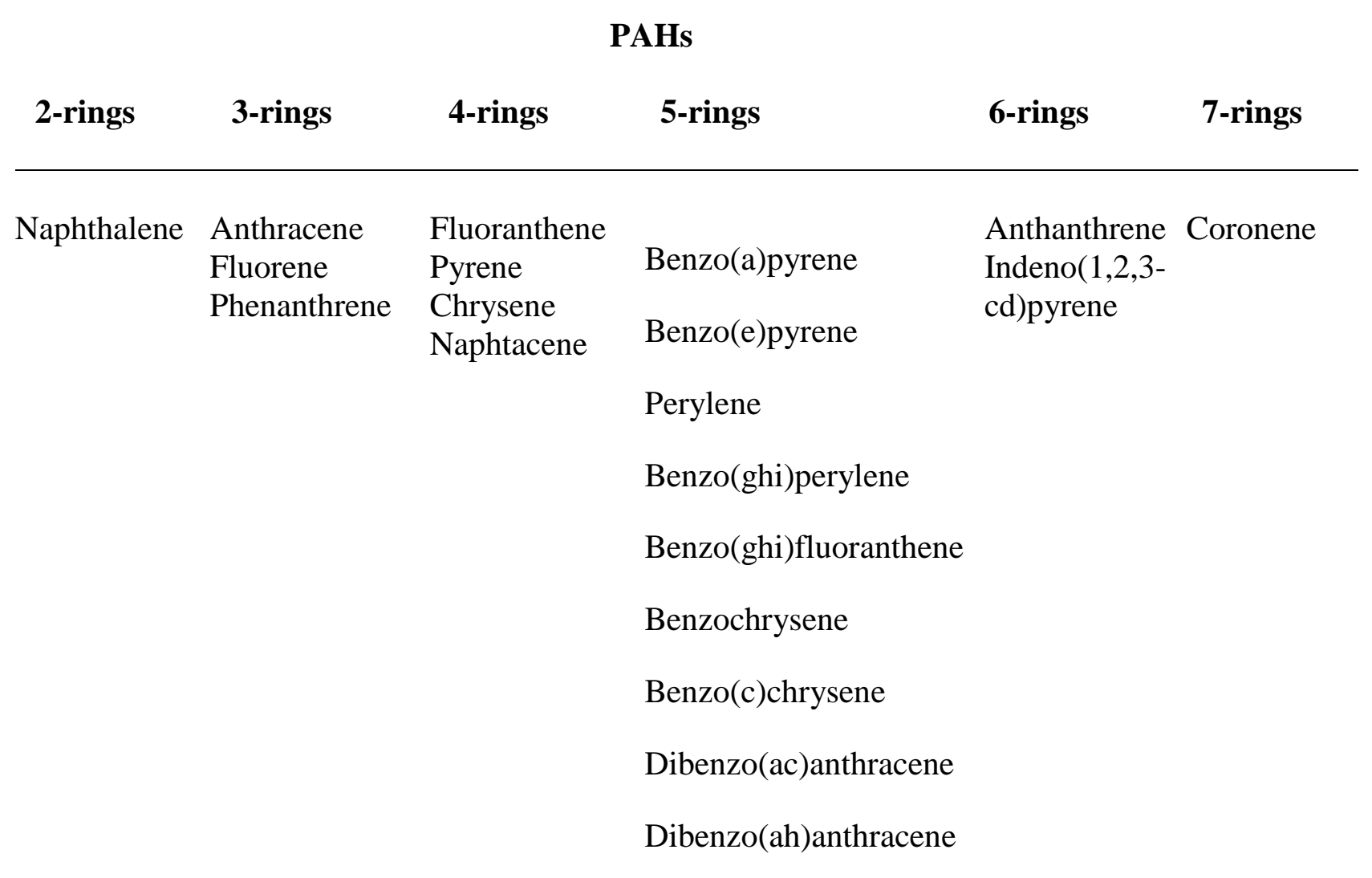

\section{DIMERS of PAHs}

2-rings $\quad$ 3-rings $\quad$ 4-rings $\quad$-rings

Anthracene Pyrene

Benzo(a)pyrene

Perylene

Benzo(ghi)perylene 
Naphthalene

Fluorene

Anthracene

Phenanthrene

Fluoranthene

Pyrene

Chrysene

Naphthacene

Perylene

Benzo(ghi)perylene

Benzo(a)pyrene

Benzo(e)pyrene

Benzo(ghi)fluoranthene

Benzo(b)chrysene

Benzo(c)chrysene

Dibenzo(ac)anthracene

Dibenzo(ah)anthracene

Coronene

Anthanthrene

Indeno(1,2,3-cd)pyrene
Karcher et al. ${ }^{29}$

Karcher et al. ${ }^{29}$

Karcher et al. ${ }^{37}$

Karcher et al. ${ }^{37}$

Karcher et al. ${ }^{37}$

Karcher et al. ${ }^{37}$

Karcher et al. ${ }^{37}$

Karcher et al. ${ }^{29}$

Karcher et al. ${ }^{29}$

Karcher et al. ${ }^{37}$

Karcher et al. ${ }^{37}$

Karcher et al. ${ }^{37}$

Karcher et al. ${ }^{37}$

Karcher et al. ${ }^{37}$

Karcher et al. ${ }^{37}$

Karcher et al. ${ }^{37}$

Karcher et al. ${ }^{37}$

Karcher et al. ${ }^{29}$

Karcher et al. ${ }^{37}$

Karcher et al. ${ }^{37}$
Orain et al. ${ }^{53}$

Karcher et al. ${ }^{29}$

Cignoli et al. ${ }^{54}$

Cignoli et al. ${ }^{54}$

Ledier $^{55}$

Cignoli et al. ${ }^{54}$

Kirsch et al. ${ }^{56}$

Karcher et al. ${ }^{29}$

Cignoli et al. ${ }^{54}$

Cignoli et al. ${ }^{54}$

Cignoli et al. ${ }^{54}$

Karcher et al. ${ }^{37}$

Karcher et al. ${ }^{37}$

Karcher et al. ${ }^{37}$

Karcher et al. ${ }^{37}$

Karcher et al. ${ }^{37}$

Karcher et al. ${ }^{37}$

Cignoli et al. ${ }^{54}$

Karcher et al. ${ }^{37}$

Karcher et al. ${ }^{37}$

Table 2: The corresponding references for the absorption and emission spectra used in our model. 
670

671

672

673

674

675

676

677

\begin{tabular}{ccc} 
Excimer & Excimer Peak Fluorescence $\left(\mathbf{c m}^{\mathbf{- 1}}\right)$ & Binding Energy $\mathbf{D}_{\mathbf{e}}\left(\mathbf{c m}^{\mathbf{- 1}}\right)$ \\
\hline Anthracene & $22220^{\mathrm{a}}$ & $2200^{\mathrm{a}}$ \\
& $20920^{\mathrm{b}}$ & $6890^{\mathrm{b}}$ \\
Pyrene & $20530^{\mathrm{c}}$ & $6350^{\mathrm{c}}$ \\
& $20750^{\mathrm{d}}$ & $6130^{\mathrm{d}}$ \\
& & $5860^{\mathrm{e}}$ \\
Benzo(a)pyrene & $19460^{\mathrm{b}}$ & $8040^{\mathrm{b}}$ \\
& & $5360^{\mathrm{d}}$ \\
Perylene & $17300^{\mathrm{b}}$ & $5750^{\mathrm{b}}$ \\
Benzo(ghi)perylene & $19840^{\mathrm{d}}$ & $6060^{\mathrm{b}}$ \\
& & $6850^{\mathrm{d}}$ \\
\hline
\end{tabular}

678

679

Table. 3: Fluorescence and binding energy of several excimers.

680

(a) Chakraborty et al. ${ }^{57}$, (b) Azumi et al. ${ }^{49}$, (c) Birks et al. ${ }^{58}$,

681

(d) Birks et al. ${ }^{59}$, (e) Birks et al. ${ }^{60}$

682 
684

685

686

687

688

689

690

691

692

693

694

695

696

697

698

699

700

701

702

703

704

705

706

707

708

709

710

711

712

713

714

715

716

717

718

719

720

721

722

723

724

725

726

727

728

729

1 R. F. Service, Study Fingers Soot as a Major Player in Global Warming, Science, 2008, 319, 1745-1745.

2 A. D'Anna, Combustion-formed nanoparticles, Proc. Combust. Inst., 2009, 32, 593-613.

3 A. E. Karatas and Ö. L. Gülder, Soot formation in high pressure laminar diffusion flames, Prog. Energy Combust. Sci., 2012, 38, 818-845.

4 M. Frenklach and H. Wang, Detailed modeling of soot particle nucleation and growth, Symp. Int. Combust., 1991, 23, 1559-1566.

5 H. Wang, Formation of nascent soot and other condensed-phase materials in flames, Proc. Combust. Inst., 2011, 33, 41-67.

6 H. Sabbah, L. Biennier, S. J. Klippenstein, I. R. Sims and B. R. Rowe, Exploring the Role of PAHs in the Formation of Soot: Pyrene Dimerization, J. Phys. Chem. Lett., 2010, 1 , 2962-2967.

7 T. S. Totton, A. J. Misquitta and M. Kraft, A quantitative study of the clustering of polycyclic aromatic hydrocarbons at high temperatures, Phys. Chem. Chem. Phys., 2012, 14, 4081-4094.

8 A. Violi, A. Kubota, T. N. Truong, W. J. Pitz, C. K. Westbrook and A. F. Sarofim, A fully integrated kinetic monte carlo/molecular dynamics approach for the simulation of soot precursor growth, Proc. Combust. Inst., 2002, 29, 2343-2349.

9 B. Zhao, Z. Yang, Z. Li, M. V. Johnston and H. Wang, Particle size distribution function of incipient soot in laminar premixed ethylene flames: effect of flame temperature, Proc. Combust. Inst., 2005, 30, 1441-1448.

$10 \mathrm{H}$. Wang, Formation of nascent soot and other condensed-phase materials in flames, Proc. Combust. Inst., 2011, 33, 41-67.

11 M. R. Kholghy, A. Veshkini and M. J. Thomson, The core-shell internal nanostructure of soot - A criterion to model soot maturity, Carbon, 2016, 100, 508-536.

12 M. Alfè, B. Apicella, R. Barbella, J. N. Rouzaud, A. Tregrossi and A. Ciajolo, Structureproperty relationship in nanostructures of young and mature soot in premixed flames, Proc. Combust. Inst., 2009, 32, 697-704.

13R. Stirn, T. G. Baquet, S. Kanjarkar, W. Meier, K. P. Geigle, H. H. Grotheer, C. Wahl and M. Aigner, Comparison of Particle Size Measurements with Laser-Induced Incandescence, Mass Spectroscopy, and Scanning Mobility Particle Sizing in a Laminar Premixed Ethylene/Air Flame, Combust. Sci. Technol., 2009, 181, 329-349.

14P. D. Teini, D. M. A. Karwat and A. Atreya, Observations of nascent soot: Molecular deposition and particle morphology, Combust. Flame, 2011, 158, 2045-2055.

15 N. A. Eaves, S. B. Dworkin and M. J. Thomson, The importance of reversibility in modeling soot nucleation and condensation processes, Proc. Combust. Inst., 2015, 35, 1787-1794.

16B. L. Wersborg, L. K. Fox and J. B. Howard, Soot concentration and absorption coefficient in a low-pressure flame, Combust. Flame, 1975, 24, 1-10.

17 J. D. Herdman and J. H. Miller, Intermolecular Potential Calculations for Polynuclear Aromatic Hydrocarbon Clusters, J. Phys. Chem. A, 2008, 112, 6249-6256.

18C. A. Schuetz and M. Frenklach, Nucleation of soot: Molecular dynamics simulations of pyrene dimerization, Proc. Combust. Inst., 2002, 29, 2307-2314.

19 N. A. Eaves, S. B. Dworkin and M. J. Thomson, Assessing relative contributions of PAHs to soot mass by reversible heterogeneous nucleation and condensation, Proc. Combust. Inst., 2017, 36, 935-945. 
20D. Aubagnac-Karkar, A. El Bakali and P. Desgroux, Soot particles inception and PAH condensation modelling applied in a soot model utilizing a sectional method, Combust. Flame, 2018, 189, 190-206.

21 M. R. Kholghy, G. A. Kelesidis and S. E. Pratsinis, Reactive polycyclic aromatic hydrocarbon dimerization drives soot nucleation, Phys. Chem. Chem. Phys., 2018, 20, 10926-10938.

22 K. O. Johansson, M. P. Head-Gordon, P. E. Schrader, K. R. Wilson and H. A. Michelsen, Resonance-stabilized hydrocarbon-radical chain reactions may explain soot inception and growth, Science, 2018, 361, 997-1000.

23 J. B. Birks, Excimers, Rep. Prog. Phys., 1975, 38, 903.

24 Y. Numata, T. Nirasawa and I. Suzuka, Excited states of pyrene excimer observed by photodissociation spectroscopy in a supersonic jet, J. Photochem. Photobiol. Chem., 2010, 209, 27-31.

25H. Saigusa and E. C. Lim, Excimer Formation in van der Waals Dimers and Clusters of Aromatic Molecules, Acc. Chem. Res., 1996, 29, 171-178.

26F. Beretta, V. Cincotti, A. D'Alessio and P. Menna, Ultraviolet and visible fluorescence in the fuel pyrolysis regions of gaseous diffusion flames, Combust. Flame, 1985, 61, 211218.

27D. S. Coe, B. S. Haynes and J. I. Steinfeld, Identification of a source of argon-ion-laser excited fluorescence in sooting flames, Combust. Flame, 1981, 43, 211-214.

28 S. Bejaoui, X. Mercier, P. Desgroux and E. Therssen, Laser induced fluorescence spectroscopy of aromatic species produced in atmospheric sooting flames using UV and visible excitation wavelengths, Combust. Flame, 2014, 161, 2479-2491.

29 W. Karcher, S. Ellison, M. Ewald, P. Garrigues, E. Gevers and J. Jacob, Spectral atlas of polycyclic aromatic compounds Vol.2.

30J. Wu, K. H. Song, T. Litzinger, S.-Y. Lee, R. Santoro and M. Linevsky, Reduction of PAH and soot in premixed ethylene-air flames by addition of dimethyl ether, Combust. Sci. Technol., 2006, 178, 837-863.

31 J. H. Miller, Aromatic excimers: evidence for polynuclear aromatic hydrocarbon condensation in flames, Proc. Combust. Inst., 2005, 30, 1381-1388.

32 M. Sirignano, A. Collina, M. Commodo, P. Minutolo and A. D'Anna, Detection of aromatic hydrocarbons and incipient particles in an opposed-flow flame of ethylene by spectral and time-resolved laser induced emission spectroscopy, Combust. Flame, 2012, 159, 1663-1669.

33C. Irimiea, A. Faccinetto, X. Mercier, I.-K. Ortega, N. Nuns, E. Therssen, P. Desgroux and C. Focsa, Unveiling trends in soot nucleation and growth: When secondary ion mass spectrometry meets statistical analysis, Carbon, , DOI:10.1016/j.carbon.2018.12.015.

34C. Irimiea, A. Faccinetto, Y. Carpentier, I.-K. Ortega, N. Nuns, E. Therssen, P. Desgroux and C. Focsa, A comprehensive protocol for chemical analysis of flame combustion emissions by secondary ion mass spectrometry, Rapid Commun. Mass Spectrom., 2018, 32, 1015-1025.

35R. L. Vander Wal, K. A. Jensen and M. Y. Choi, Simultaneous laser-induced emission of soot and polycyclic aromatic hydrocarbons within a gas-jet diffusion flame, Combust. Flame, 1997, 109, 399-414.

36I. B. Berlman, Handbook of Fluorescence Spectra of Aromatic Molecules, Academic Press second edition, New York, 1971.

37 W. Karcher, R. J. Fordham, J. J. Dubois, P. G. J. M. Glaude and J. a. M. Ligthart, Spectral atlas of polycyclic aromatic compounds Vol.1. 
38 K. Nakagawa, Y. Numata, H. Ishino, D. Tanaka, T. Kobayashi and E. Tokunaga, Excimer Luminescence From Nonresonantly Excited Pyrene and Perylene Molecules in Solution, $J$. Phys. Chem. A, 2013, 117, 11449-11455.

39S. E. Stein and A. Fahr, High-temperature stabilities of hydrocarbons, J. Phys. Chem., 1985, 89, 3714-3725.

40J. S. Lowe, J. Y. W. Lai, P. Elvati and A. Violi, Towards a predictive model for polycyclic aromatic hydrocarbon dimerization propensity, Proc. Combust. Inst., 2015, 35, 1827-1832.

41 T. Mouton, X. Mercier and P. Desgroux, Isomer discrimination of PAHs formed in sooting flames by jet-cooled laser-induced fluorescence: application to the measurement of pyrene and fluoranthene, Appl. Phys. B, 2016, 122, 123-139.

42 H. Grosch, Z. Sárossy, H. Egsgaard and A. Fateev, UV absorption cross-sections of phenol and naphthalene at temperatures up to $500^{\circ} \mathrm{C}$, J. Quant. Spectrosc. Radiat. Transf., 2015, 156, 17-23.

43 A. Thöny and M. J. Rossi, Gas-phase UV spectroscopy of anthracene, xanthone, pyrene, 1bromopyrene and 1,2,4-trichlorobenzene at elevated temperatures, J. Photochem. Photobiol. A, 1997, 104, 25-33.

44H. Saigusa and E. C. Lim, Pump-probe fluorescence studies of excimer formation and dissociation for the van der Waals dimer of fluorene, J. Phys. Chem., 1991, 95, 2364-2370.

45F. M. Winnik, Photophysics of preassociated pyrenes in aqueous polymer solutions and in other organized media, Chem. Rev., 1993, 93, 587-614.

$46 \mathrm{H}$. Sumi, Two kinds of excimers in $\alpha$-perylene and pyrene crystals: Origin of Y and V emissions, Chem. Phys., 1989, 130, 433-449.

47 O. A. Khakhel, Absorption Spectra of Pyrene Aggregates in Saturated Solutions, J. Appl. Spectrosc., 2001, 68, 280-286.

48 M. Kühni, C. Morin and P. Guibert, Fluoranthene laser-induced fluorescence at elevated temperatures and pressures: implications for temperature-imaging diagnostics, Appl. Phys. B Lasers Opt., 2011, 102, 659-671.

49T. Azumi and S. P. McGlynn, Energy of Excimer Luminescence. I. A Reconsideration of Excimer Processes, J. Chem. Phys., 1964, 41, 3131-3138.

50F. Ossler, T. Metz and M. Aldén, Picosecond laser-induced fluorescence from gas-phase polycyclic aromatic hydrocarbons at elevated temperatures. II. Flame-seeding measurements, Appl. Phys. B Lasers Opt., 2001, 72, 479-489.

51R. A. Dobbins, R. A. Fletcher and H. C. Chang, The evolution of soot precursor particles in a diffusion flame, Combust. Flame, 1998, 115, 285-298.

52 B. D. Adamson, S. A. Skeen, M. Ahmed and N. Hansen, Detection of Aliphatically Bridged Multi-Core Polycyclic Aromatic Hydrocarbons in Sooting Flames with Atmospheric-Sampling High-Resolution Tandem Mass Spectrometry, J. Phys. Chem. A, 2018, 122, 9338-9349.

53 M. Orain, P. Baranger, B. Rossow and F. Grisch, Fluorescence spectroscopy of naphthalene at high temperatures and pressures: Implications for fuel-concentration measurements, Appl. Phys. B Lasers Opt., 2011, 102, 163-172.

54F. Cignoli, G. Zizak, S. Benecchi and D. Tencalla, Atlas of fluorescence of spectra of aromatic hydrocarbons, Editorial group \& Graphic design: S. De Iuliis, D. Ferretti, 1992.

55C. Ledier, PhD Thesis, University Paris Sud, 2011.

56B. A. Kirsch and J. D. Winefordner, Electrothermal vaporization and laser-induced fluorescence for screening of polyaromatic hydrocarbons, Anal. Chem., 1987, 59, 18741879.

57T. Chakraborty and E. C. Lim, Study of van der Waals clusters of anthracene by laserinduced fluorescence in a supersonic jet: evidence for two structurally different dimers, $J$. Phys. Chem., 1993, 97, 11151-11153. 
828 58J. B. Birks and L. G. Christophorou, Excimer Fluorescence of Aromatic Hydrocarbons in $829 \quad$ Solution, Nature, 1962, 194, 442-444.

830 59J. B. Birks and L. G. Christophorou, Resonance Interactions of Fluorescent Organic $831 \quad$ Molecules in Solution, Nature, 1962, 196, 33-35.

832 60J. B. Birks, D. J. Dyson and I. H. Munro, 'Excimer' fluorescence II. Lifetime studies of 833

834 pyrene solutions, Proc R Soc Lond A, 1963, 275, 575-588. 